\title{
Format organisationnel et violence d'état : le cas de l'Ulster Defence Regiment en Irlande du Nord
}

\section{Anne Mandeville}

\section{(2) OpenEdition \\ Journals}

Édition électronique

URL : http://journals.openedition.org/conflits/221

DOI : $10.4000 /$ conflits.221

ISSN : $1777-5345$

Éditeur :

CCLS - Centre d'études sur les conflits lilberté et sécurité, L'Harmattan

Édition imprimée

Date de publication : 15 mai 1993

ISSN : 1157-996X

\section{Référence électronique}

Anne Mandeville, «Format organisationnel et violence d'état : le cas de l'Ulster Defence Regiment en Irlande du Nord », Cultures \& Conflits [En ligne], 09-10 | printemps-été 1993, mis en ligne le 04 mars 2005, consulté le 30 mars 2021. URL : http://journals.openedition.org/conflits/221 ; DOI : https:// doi.org/10.4000/conflits.221

Ce document a été généré automatiquement le 30 mars 2021 


\title{
Format organisationnel et violence d'état : le cas de l'Ulster Defence Regiment en Irlande du Nord
}

\author{
Anne Mandeville
}

L'un des aspects primordiaux de la "politique du maintien de l'ordre" est le choix du "format" des forces de l'ordre. Samuel Finer utilisait ce concept à propos des forces armées ${ }^{1}$. Selon lui, "dans sa définition la plus étroite et la plus explicite, le format signifie simplement le principe gouvernant l'emploi des forces. On s'intéresse ainsi au fait qu'elles soient indigènes ou étrangères, payées ou non payées, ad hoc ou permanentes"2. A partir de cette définition de Finer, nous avons procédé, par analogie et par extension, à la création du concept de format organisationnel des forces de sécurité intérieure. On entendra ainsi les principes de composition et d'organisation des forces : locales ou nationales, professionnelles ou à temps partiel, volontaires ou de service obligatoire, civiles ou militaires, et tout ce qui se rattache à ce problème de la composition organique de la force. On peut dire en effet que le concept de format joue, dans les sociétés démocratiques, à un double niveau à savoir celui de la légitimité démocratique de l'usage de la force, et celui, technique, de l'appréciation de l'efficacité de l'usage de la force. Le problème de détermination de la force que l'on peut utiliser à bon droit pour maintenir l'ordre intérieur constitue, pour les décideurs d'un Etat démocratique, une question fondamentale; c'est ce que l'on appelle l'acceptabilité du système de maintien de l'ordre. Définie de façon simpliste, l'efficacité de la force se mesure à sa capacité de réduire le désordre (ou la violence) voire la simple menace ayant suscité la réponse de l'Etat. On peut considérer qu'il existe toujours un rapport entre le type de force choisi pour l'action et son efficacité. Dans le cadre des sociétés démocratiques, la forme que prend l'action de l'Etat face à des désordres ou à des violences internes combine, ou tente de combiner, ces deux critères de la légitimité (acceptabilité) et de l'efficacité, qui sont d'ailleurs étroitement imbriqués. Cette question se pose dans le cadre de l'une des grandes démocraties européennes: le "Royaume-Uni de Grande-Bretagne et d'Irlande du Nord" et, plus particulièrement, à travers l'étude des problèmes posés par le format du "Régiment pour la Défense de 
l'Ulster" ("Ulster Defence Regiment", ci-après UDR), force utilisée pour la sécurité intérieure en Irlande du Nord d'avril 1970 jusqu'à juillet 1992. L'examen des caractéristiques spécifiques de cette force de sécurité intérieure est particulièrement riche d'enseignements. En effet, ce régiment extrêmement controversé est l'un des rares exemples de force à avoir été créée pour le règlement d'un conflit particulier. Dans le cas précis de l'UDR, le choix du format a revêtu, à l'époque, un caractère absolument fondamental. Cette importance devait être confirmée a posteriori, mais dans un sens négatif, en raison du bilan politique désastreux qui a résulté de ce choix. En effet, le destin de cette organisation démontre, à notre sens, son échec retentissant en tant que force de sécurité intérieure, c'est-à-dire en tant qu'instrument de l'Etat britannique pour le règlement $d u$ conflit en Irlande du Nord, étant donné son incapacité à en faire baisser l'intensité. Cet échec, qui s'intègre bien entendu dans un bilan général de l'action des forces de l'ordre en Irlande du Nord, est aussi un échec spécifique de l'UDR. Il se manifeste essentiellement par un problème d'identité, à la fois créé et intensifié par le choix d'un format inadapté au contexte conflictuel nordirlandais. On doit en effet considérer qu'avant même d'être un instrument du pouvoir d'Etat, une force est une organisation en interaction permanente avec un environnement particulier, à savoir le type de conflit dans lequel elle doit agir, mais aussi la structure du système de maintien de l'ordre dont elle est partie intégrante. Cette structure comprend principalement, en dehors des forces de l'ordre proprement dites, les autorités et les règles gouvernant l'usage des forces. Le choix du format s'analyse donc comme une décision politique majeure : il est bien évident que le type d'organisation choisi va influencer les rapports que celle-ci est susceptible d'entretenir avec son environnement. Cette décision politique est particulièrement importante dans le cas où cet environnement est fondamentalement conflictuel. Du fait d'un mauvais choix du format organisationnel, l'action de l'Etat non seulement ne restaure pas l'ordre mais est susceptible de créer du désordre et d'engendrer de la violence dont nous verrons, dans le cas nord-irlandais, qu'elle peut être à la fois physique et symbolique.

\section{LE CHOIX DU FORMAT DE L'ULSTER DEFENCE REGIMENT}

3 En janvier 1970, le Gouvernement britannique créait une nouvelle force de sécurité interne : le Régiment pour la Défense de l'Ulster. Cette décision intervenait dans un contexte particulier : les très violents affrontements de l'été 1969 entre Catholiques et Protestants mais aussi entre Catholiques et forces de l'ordre, avaient été d'une telle férocité qu'ils avaient nécessité l'envoi de l'armée régulière pour une mission de maintien de la paix. Les rapports des enquêtes officielles ${ }^{3}$ avaient stigmatisé le système politique nord-irlandais qu'ils rendaient largement responsable des troubles, mais aussi et surtout le système de maintien de l'ordre nord-irlandais, présenté comme totalement non-professionnel, et surtout extrêmement partial. Ces critiques visaient la Police de l'Irlande du Nord, la "Royal Ulster Constabulary" (ci-après RUC), mais aussi et surtout la police dite "spéciale", nommée "Ulster Special Constabulary" (ci-après USC). Le rapport Hunt, notamment, recommandait en tout premier lieu la dissolution de l'USC. Celle-ci était devenue un "anti-modèle". Pour situer l'USC il nous faut remonter aux origines de sa création, à savoir le tournant du siècle, au moment de la campagne 
pour l'autonomie de l'Irlande. La résistance protestante prit la forme d'une puissante milice, l'"Ulster Volunteer Force", destinée à empêcher par la force toute velléité de séparation de l'Irlande avec la Grande-Bretagne. La première guerre mondiale vint conforter ces "Unionistes" dans leur sentiment d'appartenance au Royaume-Uni (de nombreux protestants se battirent en effet aux côtés des Britanniques), et il s'ensuivit une véritable guerre avec les nationalistes irlandais qui déboucha finalement sur la partition de l'Irlande en 1920 et sur la création d'un gouvernement autonome en Irlande du Nord. La partition elle-même, quoique satisfaisant les Unionistes protestants puisqu'elle se fit selon des critères "ethniques" (le nord protestant et le sud catholique), allait déclencher chez eux une véritable "mentalité d'assiégés", et une sorte d'obsession de l'invasion par Dublin. Ce sentiment d'insécurité était renforcé par la présence d'une forte minorité catholique sur le territoire nord-irlandais, ainsi que par la conviction que le gouvernement britannique ne pouvait assurer la sécurité intérieure de l'Ulster. Pour tenter d'y remédier, ils eurent recours, avec l'accord du gouvernement britannique, à l'institution britannique séculaire de la "Special Constabulary", c'est-àdire à une forme de milice citoyenne. Le format de l'USC était particulièrement intéressant. C'était une force de milice, organisée sur la base du comté, mais théoriquement sans déploiement dans les zones catholiques. Armés et volontaires, ses membres étaient utilisés à temps partiel, sous le contrôle théorique de la RUC. C'était donc une force civile ${ }^{4}$. Le recrutement des "Specials" fut immédiatement noyauté par les anciens de l'Ulster Volunteer Force, avec la plus grande complicité des autorités unionistes. L'USC conserva tout au long de son existence l'aspect d'une force entièrement protestante, coutumière d'une attitude toujours discriminatoire au détriment de la minorité catholique. En 1969, les critiques les plus sévères seront dirigées contre le gouvernement britannique accusé d'avoir laissé, non sans une certaine complaisance, se développer cette véritable "armée de l'intérieur" assimilant sa mission de défense de la communauté à la défense par la force de l'Etat protestant, au mépris des droits les plus élémentaires de la minorité. C'est donc pour succéder à cette force à la fois non-professionnelle et partiale que devait naître l'UDR. Face à l'"anti-modèle" que constituait l'USC, les qualités requises pour la nouvelle force étaient donc claires : impartialité et professionnalisme. La dissolution de l'USC en plein conflit intercommunautaire créait un vide. Selon le rapport Hunt, l'USC remplissait en effet à la fois des "tâches militaires et des tâches policières". L'idée était de distinguer ces deux rôles et de créer, en remplacement, "deux forces séparées". "L'une serait une force de police de réserve, pouvant assister la RUC pour la police normale. L'autre serait une force à temps partiel recrutée localement, sous le contrôle du général commandant en Irlande du Nord, pouvant assister les forces militaires régulières dans des tâches purement militaires"5. Cette dernière force était précisément l'UDR, dont l'utilisation était d'autre part exclue pour le contrôle de foule en zone urbaine. Le texte de loi portant création de l'UDR précisait qu'il était mis sur pied une force militaire à temps partiel, recrutée localement ${ }^{6}$. Cependant, les membres de l'UDR, quoique relevant des forces armées de Sa Majesté, n'étaient susceptibles de servir qu'en Irlande du Nord. Le gouvernement britannique créait donc, quoique l'expression soit en ellemême assez paradoxale, un régiment de milice. Ainsi sont résumés les deux problèmes fondamentaux, on pourrait presque dire les tares de naissance, de l'UDR. Le premier peut être discerné dans une sorte de curieux paradoxe : une force de milice est dissoute dans l'opprobre général. Que choisit-on pour lui succéder? une autre force de milice. Dans le contexte sectaire, cette décision devait avoir des conséquences fondamentales 
sur le plan de l'identité politique de la nouvelle force. Le second réside dans cette étrange répartition des tâches en deux catégories : la "police normale" et les "tâches purement militaires". On peut faire à ce sujet plusieurs remarques : tout d'abord, au moment de la création de l'UDR, on peut considérer qu'il n'y a déjà plus en Irlande du Nord de "police normale". Même aujourd'hui, en 1993, un policier ne peut délivrer une simple contravention dans un quartier catholique sans être accompagné d'au moins six militaires. En 1969, l'armée assumait la responsabilité de la totalité de la "sécurité interne" en Irlande du Nord. Quelles étaient donc ces tâches "purement militaires" auxquelles faisait sobrement allusion le texte? Cette obscurité dans la définition des tâches, plus tard reconnue comme l'une des caractéristiques de la "politique de sécurité" britannique en Irlande du Nord, est à la base de l'autre problème majeur de l'UDR à savoir son identité en tant que force de maintien de l'ordre. Les membres de l'UDR se sont toujours présentés comme membres "à part entière" de l'armée britannique. Cependant, en dehors précisément de cette qualification de régiment, ainsi que de la sujétion de l'UDR aux règlements militaires, les ressemblances avec les autres unités de l'armée sont minces. La différence essentielle réside évidemment dans la composition du régiment dont on décida, en 1969, qu'il recruterait presque exclusivement des volontaires à temps partiel ${ }^{7}$. Le "modèle militaire" de formation de l'UDR s'exprimait essentiellement dans l'encadrement: tous les officiers supérieurs provenaient de l'armée régulière (c'est-à-dire qu'ils n'étaient pas des locaux). Théoriquement, selon des critères militaires, tous les bataillons dont était composé l'UDR auraient dû présenter une organisation identique ; en réalité, seuls certains des onze bataillons ont jamais été capables de se doter d'une organisation cohérente et réglementaire. Ceci nous amène à une autre caractéristique de l'organisation de l'UDR, elle aussi héritée de la milice. La loi faisant référence à un recrutement local, on répartit la force en comtés (sur le modèle de l'USC...). Ceci en fit une institution extrêmement dépendante de la situation locale, étant donné la règle absolue de recrutement des hommes dans leur "communauté". Cette règle a toujours été maintenue malgré certaines modifications ${ }^{8}$. Tous les soldats travaillaient donc forcément à l'endroit où ils vivaient. Cette particularité a empêché l'émergence d'un modèle d'organisation "rationnelle" des bataillons. La plupart des officiers britanniques ont toujours trouvé cette organisation extrêmement inefficace, considérant cette irrationalité comme l'un des exemples du caractère politiquement tabou de tout ce qui touchait à l'UDR. Le statu quo est donc resté jusqu'à la fin la règle en cette matière. Cependant la toute récente décision de fusion entre l'UDR et un autre régiment de l'armée britannique semble constituer un pas en direction de l'adoption de cette logique militaire. Elle ménage cependant encore, et ce n'est pas le moindre de ses paradoxes, la composante à temps partiel de l'UDR, à laquelle est toujours resté identifié le régiment dans l'opinion publique, que celle-ci soit catholique ou protestante, et ceci malgré l'importance croissante du nombre de "professionnels" (i.e. de permanents). La règle du localisme s'appliquait aussi aux missions de l'UDR. Le régiment, et c'est encore une différence essentielle, peut-être la plus fondamentale, avec les autres régiments de l'armée, ne pouvait être employé qu'en Irlande du Nord, et uniquement pour la sécurité intérieure. Le choix du format de milice, antithèse de la force professionnelle, constitue donc un véritable paradoxe, qui apparaît immédiatement si l'on procède à une comparaison entre les caractéristiques de l'UDR et de l'USC. Ceci est d'autant plus gênant que les conditions dans lesquelles fut prévu le recrutement de la nouvelle force pouvaient faire douter soit de la bonne foi, soit de la 
clairvoyance du gouvernement. Roy Hattersley, Secrétaire d'Etat à la Défense, signifiait, en présentant l'UDR au Parlement de Westminster, qu'il serait indispensable de s'appuyer sur l'USC. Cette politique est également affirmée sans ambiguïté dans le texte de création de l'UDR'. Comment comprendre cette attitude pour le moins équivoque des autorités britanniques?

\section{LES DETERMINANTS DE LA DECISION DU GOUVERNEMENT}

Le choix du format dépend, rappelons-le, de l'interprétation par les autorités politiques des critères fondamentaux d'acceptabilité et d'efficacité. Cette interprétation est bien sûr conditionnée par un certain nombre de facteurs. Nous avons retenu deux dimensions particulièrement importantes dans le cas du Royaume-Uni : la place des traditions et le poids du "système" de maintien de l'ordre. Le "format" de la milice est un modèle britannique traditionnel dont les "Specials" (ou "Special Constables") ne sont que l'une des applications. Leur reconnaissance dans un texte de loi date de $1662^{10}$, mais on peut faire remonter leur conception à l'institution médiévale du "posse comitatus", un pouvoir que détenait l'"autorité locale" (en général le shérif) de convoquer les citoyens pour le maintien de l'ordre. Cette pratique est plus largement intégrée dans une conception générale du maintien de l'ordre que nous qualifierons de communautaire ou citoyenne (au sens anglais du terme, c'est-à-dire par opposition à l'Etat). En effet, selon la doctrine et la pratique constitutionnelle britanniques, le citoyen fait lui-même la police. C'est ainsi que s'explique la très longue tradition d'utilisation de la milice dans le maintien de l'ordre ; enrôlés en général à l'initiative des autorités locales, les "Specials" pouvaient aussi relever d'une sorte de "mobilisation nationale", en cas de situation exceptionnelle ${ }^{11}$. C'est également la même conception qui explique l'existence, historiquement récurrente dans le Royaume-Uni, d'associations de citoyens pour la "défense de la paix publique" ${ }^{12}$. En 1839, on en dénombrait en Angleterre pas moins de $500^{13}$.

Ce que l'historien Radzinowicz appelle "the spirit of self-help"14 se perpétue dans la doctrine contemporaine du "policier-citoyen", fer de lance de la doctrine policière dite "démocratique", du Royaume-Uni. La persistance contemporaine de l'institution des "Specials", voire sa réactivation très récente par le gouvernement britannique de John Major ${ }^{15}$, est une illustration supplémentaire de la puissance de cette philosophie et de ce modèle de milice, légitimé par une certaine conception "commmunautaire" de la démocratie. Dans ce sens, l'Irlande n'est donc pas du tout un "cas à part", une aberration, comme on veut trop souvent le faire croire $^{16}$. On peut à cet égard méditer sur le fameux "Bill of Rights" de 1689, la si célébrée charte parlementaire anglaise qui stipule dans son paragraphe 7 que "les sujets protestants peuvent avoir pour leur défense des armes conformes à leur condition et permises par la loi" ${ }^{17}$. Ce qu'implique donc l'existence de cette forte tradition, considérée au Royaume-Uni comme un signe de démocratie, c'est la légitimité politique dont jouit dans ce pays le modèle de milice. Ce qui est donc condamné dans l'USC en 1969 n'est pas du tout son format, c'est la mauvaise utilisation qu'en ont fait des Protestants plus soucieux d'une imposition par la violence des valeurs de l'Etat protestant que du maintien de la paix publique. La conception à la base de l'UDR est donc paradoxalement l'argument démocratique. On présente la nouvelle force comme une opportunité de mélanger et d'intégrer des 
Catholiques et des Protestants, dans un même élan démocratique pour le maintien de la paix des "bons citoyens"18. Le second facteur déterminant du choix gouvernemental est l'état du système de maintien de l'ordre ${ }^{19}$. L'une de ses caractéristiques est qu'il n'entretient pas de forces spécialisées, dans le sens où la France dispose de C.R.S et d'escadrons de Gendarmerie mobile. Cette situation, encore bien plus nette en 1969, car le système a depuis évolué, oblige à prendre en compte les options disponibles en l'absence de forces spécialisées: premièrement ce que les Britanniques appellent la police "normale" et, deuxièmement, l'institution qui peut déployer une plus grande quantité de force en cas de nécessité: l'armée. Or, de façon intéressante, le gouvernement opte en 1969 pour le "modèle militaire", qui est censé contribuer à l'effort pour transcender le caractère communautaire du maintien de l'ordre. L'adoption du "modèle militaire" doit déboucher en fait sur un processus spécifique de socialisation. Le modèle militaire propose en premier lieu un modèle d'intégration. Le gouvernement entendait ici répondre au problème de la partialité des forces de l'ordre. Le 19 novembre 1969, Denis Healey, alors ministre de la Défense, justifiait ainsi son choix devant la Chambre des Communes : "Depuis que je suis ministre de la Défense, j'ai toujours été frappé par le rôle des forces armées en Grande-Bretagne en tant qu'instrument d'intégration sociale, en tant que moyen de réunir des hommes de toutes conditions sociales et de tous les coins de Grande-Bretagne et du Commonwealth dans une unité vivante. Ceci est particulièrement vrai, par-dessus tout, des régiments nord-irlandais de l'armée britannique" ${ }^{20}$. En second lieu, le modèle militaire est un modèle professionnel. La nouvelle force est présentée comme faisant partie d'un ensemble plus vaste, celui de la profession militaire. Ceci est censé constituer une garantie contre les tendances au sectarisme que l'affiliation locale de la nouvelle force rend fortement probable aux yeux de beaucoup de critiques du "nouveau" modèle. Mais l'armée est aussi censée apporter une "technique"21. Il semble donc qu'en 1969, les Britanniques croient au modèle militaire ${ }^{22}$ ou, en tout cas, tentent $d^{\prime} y$ croire. Ces convictions sont bien sûr renforcées par la conscience de l'urgence de la situation. Tous ces éléments confèrent à l'UDR un statut qui est pour le moins ambigu. Le choix d'un format contradictoire autant en ce qui concerne l'organisation de la force et sa composition (unité militaire dite "professionnelle" mais selon une organisation de milice) qu'en ce qui concerne la définition de ses missions, vont entraîner un malaise identitaire. A la fois présentée comme bras de l'Etat mais profondément solidaire de la communauté protestante, "intégrée" dans l'armée britannique mais séparée d'elle organiquement, géographiquement et de par sa "spécificité", l'UDR est une sorte de monstre.

\section{L'INADAPTATION DU FORMAT ORGANISATIONNEL DE L'UDR A L'ENVIRONNEMENT CONFLICTUEL NORD-IRLANDAIS}

7 Elle se révèle dans le constat d'échec de l'UDR en tant que force de maintien de l'ordre, le format faisant partie des causes de l'impossibilité à obtenir impartialité et efficacité . Cette conclusion est, à notre sens, confirmée par la dissolution du régiment et son remplacement par une nouvelle force. Le format de milice n'est pas une particularité nord-irlandaise. Cependant, cette région a développé, au cours du vingtième siècle, une spécificité qui tient à la configuration du système politique. Cet état de choses rend extrêmement dangereuse l'utilisation du format communautaire de milice pour le 
maintien de l'ordre car il s'inscrit dans un environnement non consensuel. Il serait bien sûr absurde de vouloir ici dépeindre et caractériser en quelques mots un conflit qui constitue un défi à la classification ${ }^{23}$. Nous tenterons seulement d'évoquer les aspects du conflit nord-irlandais qui permettent de comprendre la place qu'a pu y tenir l'UDR, au point d'en devenir un véritable enjeu politique. 1921 est l'année de la partition de l'Irlande, ultime résultat des affrontements irlando-irlandais et anglo-irlandais dans la foulée de la guerre d'indépendance. Bien que faisant constitutionnellement partie intégrante du Royaume-Uni, de 1921 à 1972, l'Ulster devint une sorte de petit Etat, tout en constituant ce que nous appellerons une "politie ethnique"24, à savoir un système politique ethniquement structuré, c'est-à-dire dans lequel la variable ethnique est la variable dominante; l'ethnie principale, la communauté protestante d'Ulster, cohabitant avec une forte ethnie minoritaire (les catholiques). Cette configuration particulière du politique explique l'importance d'institutions cristallisant la solidarité de l'ethnie dominante dans un système politique conçu pour elle sur mesure. Le système de maintien de l'ordre était bien sûr au coeur de ce système de domination, matérialisé par des forces comme l'UVF et l'USC, et symbolisé par leur composition ethnique ( $100 \%$ de protestants). La domination quasi-absolue de la communauté protestante se doublait d'une marginalisation tout aussi absolue de la communauté catholique minoritaire et ceci explique que la courte histoire de l'"Etat" nord-irlandais soit jalonnée de heurts violents entre elles. Ceux-ci culminent à l'été 1969, lors des rituelles célébrations protestantes de la bataille de la Boyne en 1689, victoire des Protestants de Guillaume III sur les Catholiques de Jacques II $^{25}$ et sont suivies par le déploiement massif des troupes britanniques dans les rues de Londonderry et Belfast, puis sur tout le territoire nord-irlandais qu'elles n'ont pas quitté depuis. Le conflit nord-irlandais est donc, à sa source, un conflit d'identités. Ses racines historiques profondes, qui accentuent son caractère inextricable et semblent donc rendre son règlement d'autant plus illusoire, sont renforcées, à notre sens, par la conception britannique de l'ordre politique communautaire et majoritaire. Cette conception a tendance à renforcer les particularismes, en structurant l'ordre politique en "communautés", tout en reconnaissant l'existence d'une communauté dominante, ainsi légitimée à détenir l'essentiel si ce n'est la totalité des moyens d'expression de l'autorité politique. Cette conception de l'ordre est d'ailleurs à la base de l'idée de partition. Le système de maintien de l'ordre reflète en grande partie cette conception de l'ordre politique. Or, la transformation du conflit nord-irlandais en une sorte de guerre larvée et incontrôlable a consacré la monopolisation progressive du conflit par certains acteurs : les détenteurs de la force armée, à savoir les forces de l'ordre et les organisations paramilitaires terroristes. Ceci explique qu'après la dissolution du gouvernement de l'Irlande du Nord (1972) et la marginalisation progressive de l'"espace politique" par rapport à une logique d'affrontement des forces, l'UDR, force "communautaire", soit devenu pour les protestants l'incarnation de la "défense légitime" de la solidarité communautaire. A contrario, l'UDR fut, pour la communauté catholique, le symbole de l'attitude discriminatoire de l'Etat britannique à leur égard. L'UDR constitua donc dès sa création un enjeu politique. Ce caractère irréductible de la mission de l'UDR peut être perçu à travers la symbolique de son nom : "pour la défense de l'Ulster". Une telle appellation serait impensable en France; elle est parfaitement significative dans le contexte britannique nord-irlandais. L'Ulster étant assimilée à la terre protestante, la défense de l'Ulster devient rapidement la défense de la communauté protestante. Défense contre qui ? A l'époque de la création de l'UDR, les 
critiques parlementaires ne manquèrent pas à ce sujet. Lord Hunt regretta, lors du débat législatif à la Chambre des Lords, "un nom qui est un anathème aux nombreux catholiques qui auraient été disposés à faire leur devoir civique et à s'engager (dans cette force)" ${ }^{26}$. Ces commentaires sont en eux-mêmes très clairs. Le choix du nom de la nouvelle organisation, censée remplacer le trop controversé UDR, est lui aussi révélateur. Depuis le 2 juillet 1992 en effet, l'UDR dissous a fait place au "Royal Irish Regiment", résultat de la fusion entre l'UDR et le "Royal Irish Rangers". La fusion en elle-même est symbolique : le choix des "Royal Irish Rangers", régiment qui, bien que britannique, a la particularité de recruter des deux côtés de la frontière entre la République d'Irlande et le Royaurne-Uni, permet aux politiques de déclarer que "le nouveau régiment représentera les deux traditions de l'Irlande" ${ }^{27}$. Le nom du futur régiment marque donc une rupture fondamentale avec le système de référence à l'Ulster, celui de la communauté protestante, qui avait été prédominant et si controversé en 1969. La communauté protestante a toujours fait preuve d'un attachement viscéral à l'UDR, et la première manifestation en fut évidemment l'engagement enthousiaste dans ses rangs. Au contraire, au fur et à mesure que le conflit se resserrait autour des forces armées, la méfiance originelle des Catholiques se transformait en véritable haine vis-à-vis de l'institution ( $18 \%$ de Catholiques s'engagèrent au moment de la création mais furent rapidement découragés par l'IRA d'une part, qui les considérait comme des "traitres", et par l'enrôlement massif des anciens de l'USC d'autre part). L'UDR devint donc très rapidement une force quasitotalement protestante (97\% selon les chiffres officiels de 1991). On pourrait décrire les relations de l'institution avec la communauté protestante comme étant d'ordre familial, ce qui est très visible notamment dans le mode de recrutement dominant, à savoir le bouche-à-oreille, facilité par le caractère fortement intégré du système communautaire nord-irlandais. Il était très fréquent de trouver dans un bataillon les membres d'une même famille. L'un des meilleurs exemples de la solidarité et de l'identification de la communauté protestante à "ses" défenseurs fut la véritable prise en mains par cette communauté, particulièrement par le "Parti Démocratique Unioniste" du pasteur Ian Paisley, de la défense de quatre soldats de l'UDR, les "UDR $4^{128}$, dont on avait pensé qu'ils avaient été condamnés injustement. A l'opposé, l'attitude des Catholiques (et notamment des partis politiques nationalistes) s'est toujours caractérisée par des plaintes à propos du comportement des membres de l'UDR à leur encontre, ainsi que par de constants appels à la dissolution de l'institution.

\section{L'EFFICACITE DE L'UDR EN TANT QUE FORCE DE MAINTIEN DE L'ORDRE}

9 Le choix du format de l'UDR a reposé en grande partie sur la croyance dans le bienfondé du modèle militaire, censé procurer à l'UDR le statut de force "professionnelle", à l'image de l'armée britannique. Par ce concept, on faisait allusion tout d'abord au caractère "intégrateur" de la profession, par la solidarité et l'"esprit de corps". L'armée est l'une des rares institutions qui dispose d'un fort système de valeurs et est susceptible, par là-même, de proposer un modèle alternatif d'identification. Il a cela d'essentiel qu'il dépasse les clivages politico-ethniques qui constituent précisément la base du système de valeurs nord-irlandais ${ }^{29}$. En ce sens, le processus de professionnalisation est entendu comme processus de socialisation. Or, le format de 
l'UDR est ici en contradiction avec l'objectif visé. Nous avons choisi pour l'exprimer d'évoquer le problème des "conditions de vie" et celui des effets pervers de la professionnalisation. Qu'il ait été un membre à temps partiel ou un permanent, le soldat UDR ne vivait pas en caserne. Une fois le travail terminé ou sa mission remplie, il rentrait chez lui. Il est facile de voir à quel point cet état de fait est susceptible d'influencer voire de conditionner son comportement. Le fait de se retrouver régulièrement à l'extérieur du régiment, le rendait très perméable à son environnement social et, notamment, au regard que celui-ci pouvait éventuellement porter non seulement sur lui et le monde en général mais aussi sur son engagement personnel au sein de l'UDR, ainsi que sur cette organisation. Ceci est d'autant plus important à prendre en considération que l'UDR est une institution très controversée dans le contexte politique violent et troublé que l'on connait. L'"environnement social" auquel nous faisons allusion signifie en premier lieu la famille, institution encore très importante en Irlande du $\operatorname{Nord}^{30}$, mais aussi les compagnons de travail (pour les membres à temps partiel), les voisins, les amis, etc. En clair, la "communauté locale", dont on sait qu'elle exerce une influence énorme sur le citoyen britannique mais encore plus, semble-t-il, sur l'Irlandais du Nord ${ }^{31}$. En ce sens, les "conditions de vie" sont particulièrement susceptibles d'affecter la solidarité du groupe militaire, voire la discipline. On peut aussi penser que le moral du soldat variera d'autant plus que son groupe civil d'appartenance le soutiendra ou non. Ceci se traduisait bien dans le fait que l'appartenance à l'Ulster Defence Regiment constituait pour beaucoup une "affaire de famille". Les conditions de vie jouaient également à un autre niveau. Dans ce type spécifique de conflit (lutte anti-terroriste) le soldat UDR est un acteur particulièrement visé (cible "légitime" de l'IRA), rendu très vulnérable à cause de son aller et retour permanent d'un environnement civil à un environnement militaire. Quand il était "offduty", c'est-à-dire à partir du moment où il quittait le camp militaire sans être en mission, le soldat UDR se retrouvait sans aucune protection. La preuve en est que la plupart des soldats UDR assassinés l'ont été "off-duty" (153 sur 191 de 1971 à 1991), souvent chez eux ou sur le chemin du retour. Quarante cinq anciens UDR ont été assassinés plusieurs années après avoir quitté le régiment. L'existence de cette menace permanente et tout à fait réelle affectait bien entendu la psychologie du soldat, notamment sous la forme du stress qui était l'un des problèmes les plus importants rencontrés par le régiment : pas le "battlefield stress" ou "stress in battle", bien connu des militaires ${ }^{32}$, et contre lequel on lutte avec de l'entraînement ; mais le stress général, l'angoisse qui peut se manifester à tout moment. Le taux de suicide était d'ailleurs relativement élevé à l'intérieur du régiment. Ces "conditions" particulières de vie affectaient aussi l'organisation générale du régiment : les bataillons passaient beaucoup de temps à s'occuper de la protection de leurs recrues off-duty, à surveiller leur environnement, notamment dans leur travail civil. De même, beaucoup d'énergie était investie dans la sélection et la surveillance de "planques", dans lesquelles il fallait bien souvent se résoudre à cacher les soldats et leurs familles quand ils étaient menacés ; quand il ne fallait pas tout simplement les "déplacer" définitivement hors de leur communauté lorsque l'environnement devenait trop dangereux pour leur vie. En résumé, le facteur des conditions de vie spécifiques à l'UDR était susceptible d'affecter globalement la capacité opérationnelle du régiment. Il faut également évoquer les effets pervers de la professionnalisation de l'UDR. Selon James Wilson ${ }^{33}$, le but fondamental d'une organisation est sa propre survie. Cette idée, développée abondamment par la sociologie des organisations, a été notamment appliquée à l'étude 
$\mathrm{du}$ fonctionnement des mouvements terroristes. Il nous semble que l'UDR peut constituer une étude de cas de l'application de ces théories aux forces de sécurité. Ce que nous appelons ici la "logique organisationnelle" est l'apparition d'un "système" UDR: la création d'une organisation telle que l'UDR et son orientation engendrent forcément un système d'acteurs, et d'actions. L'UDR comme toute organisation, distribuait des ressources (humaines, économiques), et du pouvoir... En tant qu'acteur social, elle pouvait avoir des intérêts ou des frustrations, de même que les acteurs qui la composaient. L'organisation doit être vue ici à la fois comme un système d'action autonome ${ }^{34}$, obéissant à ses propres règles de fonctionnement, en partie indépendantes de la contrainte d'efficacité imposée par l'environnement, mais aussi comme un organisme ouvert aux influences d'un environnement susceptible d'engendrer des modifications à l'intérieur de l'organisation. Cette dernière caractéristique est d'autant plus vraie que l'UDR faisait partie des "instruments de souveraineté", plus soumis que d'autres organisations à l'influence directe du Politique. Un exemple concret, parmi les plus intéressants, de cette "logique organisationnelle" est le problème de la carrière. La création notamment des compagnies de "permanents", et la faveur certaine dont jouissait ce mode de recrutement, ont encouragé bon nombre de membres de l'organisation à regarder l'engagement dans l'UDR comme l'entrée dans une véritable carrière professionnelle. Ce fut le cas de beaucoup de jeunes femmes, dont le recrutement fut fortement encouragé pendant les dernières années. La tendance naturelle de l'organisation a consisté ces dernières années à pousser dans le sens d'une plus forte intégration dans l'armée professionnelle. Or, cette stratégie engendrait des frustrations à plusieurs niveaux : en raison d'une part du blocage de la carrière des officiers (qui ne pouvaient pas accéder aux rangs supérieurs) mais aussi en raison des limites d'utilisation de l'UDR sur le plan militaire, avec une mission et un territoire circonscrits. On peut donc dire paradoxalement, (et l'on rejoint ici la problématique de l'effet pervers) que, selon la logique organisationnelle, le membre "professionnel" de l'UDR n'avait pas intérêt au règlement du conflit, de la même manière que l'on a pu écrire que les organisations terroristes, mues autant par leurs dynamiques internes que par les influences extérieures (notamment politiques), n'ont pas forcément pour but de "gagner" mais de continuer à jouer ${ }^{35}$.

\section{La dissolution de l'UDR : l'aveu d'un échec ?}

La décision de fusionner ce régiment avec le régiment des "Royal Irish Rangers" semble correspondre à une certaine logique : répondre, quoique tardivement, au malaise de l'UDR. Celle-ci est encore aujourd'hui présentée sous le signe de la "professionnalisation" : "L'objectif est de créer une force professionnelle hautement entrainée, sur le même modèle que tous les autres régiments d'infanterie de l'armée britannique" ${ }^{136}$. La "professionnalisation" revêt un sens différent : en effet on parle maintenant de la formation de "bons fantassins" ${ }^{37}$; on est donc loin du "devoir civique" de lutte contre le terrorisme dont parlaient Roy Hattersley et Lord Hunt en 1969. Ce changement d'orientation et de définition du professionnalisme procède du constat d'échec du format de l'UDR. La question reste cependant celle de l'interprétation de cet échec par les autorités politiques. En effet, bien des éléments restent encore obscurs et, semble-t-il, contradictoires, pour que nous soyons capables de mesurer exactement la 
signification et la portée de cette décision politique de grande importance. On doit donc se contenter de formuler des hypothèses. La question du maintien (pour le moment) de la composante à temps partiel et de son recrutement local nous paraît ainsi être en totale contradiction avec la philosophie de la fusion. Deux interprétations sont possibles. Soit la fusion n'a véritablement qu'un caractère symbolique et le statu quo reste la règle : l'échec de l'U.D.R relève en quelque sorte d'un problème de relations publiques. Ce n'est pas celui du modèle de maintien de l'ordre (on pourrait même discerner une fonction latente de la nouvelle organisation professionnelle : ouvrir des débouchés aux personnels "professionnalisés" de l'UDR). La seconde interprétation possible est que l'on est dans une stratégie d'intégration, de laïcisation politique. Cela implique à terme l'éradication de la composante à temps partiel, et l'oubli du concept même qui se trouve à la base de la création de l'UDR. L'exemple du destin du "Régiment pour la Défense de l'Ulster" montre toute l'importance qui doit être accordée par le pouvoir politique démocratique au choix des formes que doit prendre la violence de l'Etat, et particulièrement celui du format des forces de l'ordre. Nous avons ici examiné le danger de l'application du concept communautaire au système de maintien de l'ordre, à savoir l'utilisation du format de milice à l'intérieur d'un conflit intercommunautaire ou ethnique. A cet égard, les émeutes qui ont eu lieu à Los Angeles en mai 1992 peuvent être replacées dans une problématique comparable : les enquêtes menées immédiatement après les troubles n'ont fait que confirmer une fois de plus l'absence quasi-totale de légitimité non seulement de la police mais aussi du système de sécurité américain (on pense ici plus particulièrement à la Justice), à l'intérieur de la communauté noire ${ }^{38}$, ce rejet se fondant essentiellement sur la perception des institutions de sécurité comme ethniquement partiales, partialité reflétée par leur composition ainsi que leur comportement. Le deuxième problème fondamental que soulève l'étude de l'UDR est celui du contenu de la "professionnalisation" du maintien de l'ordre. Il est très clair que sur ce plan, aussi bien à l'égard des problèmes nordirlandais que des explosions de violence collective que connaît régulièrement la Grande-Bretagne depuis au moins vingt ans, la réponse des Britanniques a longtemps été essentiellement idéologique. Ils se sont néanmoins heurtés au constat de l'impossibilité du règlement de ces conflits par le simple usage d'une plus grande quantité de force, que l'on entende par là le nombre de policiers ou la puissance des matériels... En ce sens, l'expérience irlandaise et le développement d'une situation instable en Grande-Bretagne ont poussé le système de maintien de l'ordre vers une relative spécialisation ${ }^{39}$. Il n'a cependant jamais été véritablement question de "professionnalisation" du maintien de l'ordre ${ }^{40}$ au sens notamment où l'entendent les Français ${ }^{41}$. Ceci confirme à notre avis l'extraordinaire capacité de résistance des structures traditionnelles britanniques qui, alliée à celle des données conflictuelles, peut constituer l'une des explications de la relative inertie organisationnelle ${ }^{42}$ dont fait preuve, dans le domaine du maintien de l'ordre, le Royaume-Uni. 


\section{NOTES}

1. Samuel Finer, "State and Nation-building in Europe : the role of the military", in Charles Tilly (ed), The Formation of National States in Western Europe, Princeton University Press, N.J, 1975.

2. Ibidem, p.90.

3. Il s'agit d'une part du rapport Hunt (Report of the Advisory Committee on Police in Northern Ireland, Cmnd 535, H.M.S.O, Belfast, 1969), mais aussi du rapport de la Commission Cameron (Disturbances in Northern Ireland : Report of the Commission appointed by the Governor of Northern Ireland, Cmnd 532, Parlement d'Irlande du Nord, HMSO, 1969. Le rapport Scarman, publié deux ans plus tard, stigmatisera à son tour le comportement des forces de l'ordre, Report of the Tribunal of Inquiry on Violence and Civil Disturbances in Northern Ireland in 1969, Cmnd. 566, H.M.S.O, Belfast, 1972.

4. Pour plus de détails sur la structure de l'USC, voir Chris Ryder, The Ulster Defence Regiment : an Instrument of Peace ?, Methuen, London, 1991, pp.7-10.

5. Formation of the Ulster Defence Regiment, Cmnd 4188, H.M.S.O, London, 1969, p.3. 6. Ibidem. Cette décision était prise conformément aux recommandations du rapport Hunt.

7. Cette caractéristique s'est cependant estompée au fil des ans, puisque la proportion de membres permanents est passée de $10 \%$ en 1972 à 50\% en 1991. Cette évolution est amplifiée par la création au milieu des années 70 de compagnies entièrement composées de permanents. Ceci n'empêchera pas l'opinion publique de toujours considérer l'UDR comme un régiment "communautaire" et d'en garder une image fortement attachée à sa composante à temps partiel.

8. Pour plus de détails, Anne Mandeville, La professionnalisation d'une unité de maintien de l'ordre, étape de la laïcisation du système politique nord-irlandais ? Le cas de l'Ulster Defence Regiment, rapport présenté au colloque "L'Irlande : vers une société laïque ?", organisé par le Centre Universitaire d'Etudes Irlandaises, Collège des Irlandais, Paris, 22-23 Mai 1992 (A paraître).

9. "Les officiers commandant les bataillons seront des membres locaux du régiment ; pendant les premiers temps, ces postes peuvent être occupés par les actuels commandants de comté de l'Ulster Special Constabulary, lesquels sont presque tous d'anciens militaires et ont une grande expérience dans le genre de missions que la nouvelle force sera censée remplir", in Formation of the Ulster Defence Regiment, op.cit., p.4. Cette attitude fit l'objet de nombreuses controverses. Voir à ce sujet les discussions parlementaires in Hansard, 12 Novembre 1969, p.418.

10. Statute 13 \& 14, Charles II, c.12, s.15., 1662.

11. Stanley Palmer, Police and Protest in England and Ireland, Cambridge University Press, Cambridge, 1988, p.148.

12. Ces associations avaient pour but "de s'entraîner à l'exercice militaire pour pouvoir protéger leurs voisins et leurs propres biens comme le leur permet l'usage de toute méthode légale et efficace d'aide au pouvoir civil", ainsi que le déclarait l'une des nombreuses "Associations volontaires de Défense" formées lors de l'été 1780 à Londres dans la foulée des "émeutes Gordon" ("Gordon riots") de la même année (Armed Associations, Billingsgate ward, July 10,1780 (an account of the meeting and of the 
resolutions of the committee, signed by David Evans), in H.0. (Home Office papers) 42/36. Cité par Leon Radzinowicz, in History of the English Criminal Law and its Administration since 1750, Stevens, London, 1968, Vol.4, p.101.

13. Selon Adrian Schubert, "Private Initiative in Law Enforcement : associations for the prosecution of felons", in Victor Bailey, ed, Policing and Punishment in Nineteenth Century Britain, Rutgers University Press, New Brunswick, N.J, 1981, pp.25-41. Cité par Palmer, op.cit., p.148.

14. Radzinowicz, op.cit. p.100.

15. Voir par exemple à ce sujet l'article d'Alan Eastwood, "Supercops or Dad's Army ?", in Police Review, 07/06/91, pp.1160-1. Egalement : "A special relationship", Police Review, 12/07/91, pp.1428-9 ; "Specials : the Police Review survey", Police Review, 16/08/91, pp.1676-1677.

16. N'oublions pas qu'il n'y a encore qu'un peu plus d'un siècle existaient en Angleterre les "servitudes confessionnelles" (abolition en 1868).

17. Maurice Duverger, Constitutions et documents politiques, P.U.F, Paris, 1969, p.466.

18. "There will be a campaign to enrol recruits from all sections of the community" extrait de la présentation de l'UDR par Roy Hattersley, Secrétaire d'Etat à la Défense, in Hansard, 12 Novembre 1969, p.418.

19. Le mot "système" a ici le sens où l'entend James Miller dans The Organization of Life : "Les systèmes abstraits sont des ensembles de relations formelles à l'intérieur de ou entre des systèmes concrets. Leurs relations existent en tant que concepts dans l'esprit des observateurs scientifiques. Leurs sous-systèmes existent dans l'espacetemps physique et sont identifiables empiriquement." Cité par Philippe Braillard, in Théorie des systèmes et relations internationales, Ets Emile Bruylant, Bruxelles, 1977, P.54.

20. Denis Healey, cité par Chris Ryder, op.cit. p.38.

21. On peut encore ici citer à ce sujet le ministre de la Défense, qui déclarait devant une Chambre des Communes assez sceptique, à propos de la capacité des forces armées à faire du maintien de l'ordre intérieur : "Je voudrais rappeler à la Chambre et au public britannique que les forces armées ont déployé ce type de qualités en de nombreuses occasions à travers le monde durant ces vingt dernières années. La population de ce pays en a trop peu conscience", (Hansard, 13 Octobre 1969, H.M.S.O, London, 1969). 22. Pour une opinion dissonnante et cependant très respectée : Maj-General Richard Clutterbuck, "A third Force ?", in Army Quarterly, Octobre, 1973, pp.22-28. Celui-ci cite notamment, en exergue à son article, une phrase censée représenter une opinion ayant fait l'objet de nombreux débats au moment de l'explosion de violence en Irlande du Nord en 1969, et qui s'exprime ainsi : "Si l'on avait envoyé les C.R.S français dans le Bogside et à Belfast en Août 1969, tout aurait été réglé en une semaine. Il y aurait bien eu quelques os cassés, mais on n'aurait probablement pas eu de morts jusqu'à aujourd'hui".

23. On peut consulter à ce sujet les thèses devenues classiques des auteurs suivants : Arend Lijphart, "The Northern Ireland Problem : cases, theories and solutions", British Journal of Political Science, 5, pp.83-106 ; Richard Rose, Governing without Consensus : an Irish Perspective, Faber \& Faber, London, 1971 ; Liam de Paor, Divided Ulster, Penguin Books, Harmondsworth, Middlesex, 1971. Plus récemment, on lira avec profit : A.T.Q Stewart, The Roots of Conflict in Ulster, Faber \& Faber, London, 1977 ; Tom Wilson, Ulster : Conflict and Consent, Basil Blackwell Ltd, Oxford, 1989 ; Ronald Weitzer, Transforming Settler States, University of California Press, Berkeley and Los Angeles, 
1990 ; Eamonn Hughes (ed), Culture and Politics in Northern Ireland, Open University Press, Buckingham, 1991.

24. Nous empruntons ici le concept d'"ethnic polity" à Antony Smith, dans son ouvrage The Ethnic Origins of Nations, Basil Blackwell, London, 1986.

25. Ceci peut constituer l'un des éléments de la description de ce que nous considérons comme le véritable "mythe fondateur" de la communauté protestante, qui confirme la pertinence de son analyse en tant qu'ethnie.

26. Cité par Chris Ryder, op.cit. p.33.

27. Lettre d'un fonctionnaire du Secrétariat d'Etat à l'Irlande du Nord à l'auteur, 20 janvier 1992.

28. Le propre fils du pasteur Ian Paisley, Ian Paisley (jr), a rédigé un manifeste de défense de quatre membres de l'UDR qui auraient été injustement condamnés par la justice britannique. Le sigle "UDR 4" fait référence aux récents procès qui ont abouti à la remise en liberté de plusieurs irlandais catholiques eux-aussi injustement condamnés par la justice britannique à la suite de procès douteux : les "Guilford 4" et les "Birmingham 6". On peut ainsi consulter : Ian Paisley jr, The Case for a Re-trial of the UDR 4, pamphlet du Democratic Unionist Party, s.r. ; Ian Paisley jr, Reasonable Doubt : the case for the UDR 4, The Mercier Press, Dublin, 1992 ; Alderman Peter Robinson MP, Hands off the UDR, publications du Democratic Unionist Party, 1991 ; "Hands off the UDR", The New Protestant Telegraph, 07/10/89, p.l et 8.

29. Ce cadre de comportement au sein de l'Armée britannique s'articule autour de trois éléments-clés : le régiment, la "platoon", l'officier. Sur ce sujet l'ouvrage fondamental de Cynthia Enloe, Ethnic Soldiers, Penguin Books, Harmondsworth, 1980, particulièrement le chapitre 4 ("Police forces and Army-Ethnic Security Designs"). 30. John Curtice and Tony Gallagher, "The Northern Irish Dimension", in R. Jowell, S. Witherspoon, L. Brook, British Social Attitudes : The 1989 Report, Gower, Aldershot, 1989.

31. Ibidem, p.197.

32. Voir Tony Jeapes "Stress in battle ", British Army Review, 60, décembre 1980.

33. James Q. Wilson, Political Organizations, New York, Basic Books, 1973.

34. M. Crozier et E. Friedberg, L'acteur et le système. Les contraintes de l'action collective, Paris, Seuil, 1977, ainsi que M. Crozier, Le phénomène bureaucratique, Paris, Seuil, 1963.

35. A ce sujet l'excellent article de Martha Crenshaw, An Organizational Approach to the Analysis of Political Terrorism, rapport présenté au Congrès mondial de Science politique, Paris, Juillet 1985.

36. Lettre d'un fonctionnaire du Northern Ireland Office à l'auteur, 20 Janvier 1992.

37. Ibidem. L'auteur de la lettre parle de "highly professional Infantry standards".

38. Comment interpréter autrement la réponse majoritairement positive (chez les Noirs et chez les Blancs) aux questions suivantes (à propos du verdict ayant déclenché les émeutes). "A votre avis, le verdict aurait-il été différent : - s'il y avait eu des Noirs dans le jury ? - si les policiers et l'homme qu'ils ont battu avaient tous été des Blancs ? si les policiers avaient été Noirs et l'homme battu Blanc? Extraits de l'enquête de Richard Lacayo, in Time, 11 Mai 1992, p 36.

39. Voir notamment à ce sujet l'ouvrage de Gerry Northam, Shooting in the Dark : Riot Police in Britain, Faber \& Faber, London, 1988, ainsi que celui, très significatif d'une évolution des mentalités, de Peter Waddington, The Strong Arm of the Law, Open University Press, Oxford, 1991. 
40. Sur la controverse qu'a suscitée la création d'une "troisième force", Anne Mandeville : "Approche du débat sur la troisième force dans le cadre des problèmes britanniques actuels en matière de maintien de l'ordre", Toulouse, Centre d'Etudes et de Recherche sur la Police, rapport non publié, juin 1983.

41. ean-Louis Loubet del Bayle, Lucien Mandeville et Alain Picard, "Les forces de maintien de l'ordre en France", in Défense Nationale, Juillet 1977, p 59 et s. ; Dominique Monjardet, "Le maintien de l'ordre : technique et idéologie professionnelle des C.R.S", in Déviance et Société, Vol.XII, n², Juin 1988, pp.101-126 ; Dominique Monjardet, La manifestation : du côté du maintien de l'ordre, rapport présenté au Congrès annuel de l'Association Française de Science Politique, Bordeaux, 5-8 Octobre 1988.

42. Sur le concept d'inertie organisationnelle, appliqué aux forces armées, Lucien Mandeville, "Un exemple d'inertie organisationnelle : la représentation collective des intérêts des militaires en France", rapport présenté à la troisième conférence biennale de l'E.R.G.O.M.A.S, Toulouse, 2-5 Juillet 1992.

\section{RÉSUMÉS}

Cette étude se propose de faire apparaître, à travers l'examen d'une force particulière, l'Ulster Defence Regiment, la profonde insertion du système de maintien de l'ordre public dans le système politico-social britannique, à la fois sur le plan des structures, mais aussi de la culture politique. On a ainsi procédé à l'analyse des "choix" organisationnels opérés d'une part au moment de la création de cette force de maintien de l'ordre mais aussi au cours de processus de ce qu'il est convenu d'appeler sa "professionalisation". Le cas de l'Ulster Defence Regiment montre notamment l'importance de la politique de recrutement, élément fondamental de la "gestion" des forces de maintien de l'ordre intérieur, qui retentit directement sur la violence politique, que ce soit celle des forces de l'ordre elles-mêmes ou, plus largement, celle engendrée par les conflits politiques, dans lesquels les forces de l'ordre sont par définition impliquées. L'étude de cette force de maintien de l'ordre fait par ailleurs ressortir l'existence d'un espace d'autonomie, bien souvent négligé, que ce soit à l'intérieur du conflit lui-même ou à l'intérieur du système socio-politique, et donc les problèmes de gestion qui en sont la conséquence.

\section{INDEX}

Index géographique : Irlande du Nord, Royaume Uni

Mots-clés : contre-insurrection, forces paramilitaires, répression

Thèmes : Irish Republican Army (Provisional), Ulster Defence Regiment(UDF) 